\title{
Subjektive Erfahrung, intersubjektive Verständigung und Sozialität. Phänomenologische Erörterungen
}

Thomas S. Eberle

Dem Wissenschaftssystem scheint tatsächlich ein Prozess der inneren Ausdifferenzierung eigen zu sein: Es gibt immer mehr wissenschaftliche Disziplinen, Theorieund Forschungsansätze und auch immer mehr Fach- und Spezialgebiete. Oft wird diese Diagnose verknüpft mit Forderungen nach mehr Interdisziplinarität, grenzüberschreitender Zusammenarbeit, ganzheitlichem und vernetztem Denken oder gar integrativen Ansätzen. Dass sich der Trend zur Ausdifferenzierung auch bei jenen Forschungsansätzen zeigt, die sich in der deutschsprachigen Soziologie mittlerweile unter der Bezeichnung „Wissenssoziologie" versammeln, ist nicht weiter überraschend. Ebenso wenig die damit verbundene Aufforderung, eine Standortbestimmung vorzunehmen in Bezug auf die Frage „Wer oder was handelt?“

Solche Standortbestimmungen befördern bestenfalls die gegenseitige Verständigung. Indem alle Teilnehmenden in Bezug auf eine zentrale Fragestellung ihre theoretischen und vortheoretischen Prämissen reflektieren und offenlegen, wird vieles klarer, können Missverständnisse ausgeräumt werden und kann der gegenseitige Austausch bereichernd wirken. Nüchtern betrachtet ist die Chance, dass man Kolleginnen und Kollegen von der eigenen Position überzeugt, ziemlich gering - trotz aller Plausibilisierungsbemühungen. Wissenschaftssoziologisch gesehen fällt auf, dass die paradigmatische Prägung von Wissenschaftlerinnen und Wissenschaftlern berufsbiographisch meist relativ früh erfolgt, dass diese ihr Theorie- und Forschungsverständnis zwar ständig weiterentwickeln, dabei aber doch

T. S. Eberle (凶)

Universität St. Gallen, Tigerbergstrasse 2,9000 St Gallen, Schweiz

E-Mail: thomas.eberle@unisg.ch

(C) Springer Fachmedien Wiesbaden 2014

A. Poferl, N. Schröer (Hrsg.), Wer oder was handelt?,

Wissen, Kommunikation und Gesellschaft, DOI 10.1007/978-3-658-02521-2 8 
meistens innerhalb des einmal gewählten grundlegenden Forschungsansatzes verbleiben. Von dieser nüchternen Beobachtung ausgehend, versuche ich im Folgenden in aller Bescheidenheit zu erläutern, warum ich - allen gegenläufigen Trends zum Trotz - der Meinung bin, dass es für die Wissenssoziologie fruchtbar ist, die subjektive Sinnwelt des Akteurs in die Forschung miteinzubeziehen und dabei von der phänomenologischen Lebensweltanalyse von Alfred Schütz auszugehen.

Im Folgenden möchte ich zuerst kurz auf das in der Soziologie vieldiskutierte Verhältnis von Common-sense und soziologischer Perspektive eingehen. Daran anschließend erörtere ich den phänomenologischen Zugang zur subjektiven Erlebnis- und Erfahrungswelt. Daraufhin frage ich nach der Konstitution von Intersubjektivität und schließlich nach der Sozialität, also dem sozio-kulturellen Apriori des subjektiven Erlebens. Im Zuge dieser Ausführungen können verschiedene Prämissen herauskristallisiert werden, die in der gegenwärtigen qualitativen Forschungslandschaft verwendet werden. Abschließend komme ich auf die Frage zurück, warum sich Sozialwissenschaftler so gerne über ihre Prämissen streiten.

\section{Common-sense und soziologische Perspektive(n)}

Die Liste der soziologischen Distanzierungsversuche vom Common-sense ist lang. Bereits Durkheim (vgl. Durkheim 1970) empfahl in seinen Regeln der soziologischen Methode nachdrücklich, die soziologischen Begriffe von jenen des Common-sense abzugrenzen. Die szientifischen soziologischen Ansätze des 20. Jahrhunderts lebten diesem Diktum nach: Sich vom Common-sense abzugrenzen wurde gleichsam als Voraussetzung betrachtet, um einen Überlegenheitsanspruch der soziologischen Erkenntnisse geltend zu machen. Die entsprechenden Argumentationsfiguren finden wir auch heute noch in vielen soziologischen Publikationen - darauf werde ich nochmals zurückkommen.

Max Weber (vgl. Weber 1972) begründete mit seiner Verstehenden Soziologie einen alternativen Ansatz, indem er den subjektiven Sinn, den der Handelnde mit seinem Handeln verbindet, zum Angelpunkt der soziologischen Analyse machte. Zwar befasste auch er sich mit der wissenschaftlichen Begrifflichkeit und erörterte die Bildung von Durchschnitts- und Idealtypen, band diese aber mit dem Kriterium der Sinnadäquanz, die er zusätzlich zur Kausaladäquanz wissenschaftlicher Aussagen einforderte, unmittelbar an die Typisierungen des Common-sense. Der subjektive Sinn des Handelns und das Kriterium der Sinnadäquanz boten denn auch das Einfallstor für Alfred Schütz, um Webers Handlungstheorie mit der Phänomenologie zu verbinden, von Äquivokationen zu befreien und mit seiner Analyse der Strukturen der Lebenswelt (vgl. Schütz und Luckmann 1976, 1989, 2003) die Soziologie philosophisch zu begründen.

\section{Das subjektive Erleben als Ausgangspunkt und Evidenz}

Auf der Suche nach einem archimedischen Punkt der Erkenntnis versuchte Husserl bekanntlich die Kluft zwischen Rationalismus und Empirismus zu überbrücken. Er stellte sich zunächst in die rationalistische Tradition, übernahm Descartes' Methode des radikalen philosophischen Zweifels und sah im „ego cogito“ den apodiktischen Ausgangspunkt philosophischer Reflexion. Allerdings müssen die Cartesianischen Meditationen nach Husserl nochmals neu und radikaler angesetzt werden Erstens bezieht sich das „,cogito“, der Akt des Erkennens, stets auf ein „cogitatum“, ein Erkanntes, das nicht als isolierte Gegenständlichkeit gefasst werden darf. Zweitens darf das ,ego" nicht lebensweltlich verstanden, sondern muss transzendenta konzipiert werden.

Das ,ego-cogito-cogitatum“ erläutert Husserl mit Hilfe des von Franz Brentano übernommenen Intentionalitätskonzepts: Unser Bewusstsein ist immer Bewusstsein-von-etwas, es ist immer auf etwas gerichtet. Wir sehen „etwas“, hören „etwas“, fühlen „etwas“, erinnern „etwas“, fantasieren ,etwas“" usw. - das Bewusstsein ist mit anderen Worten nie leer, sondern gefüllt mit Phänomenen. Ein Phänomen ist zunächst einmal immer ein Bewusstseinsphänomen, $d$. h. es wird durch synthetische Akte des Bewusstseins konstituiert. Diese bilden den noetischen Aspekt eines Phänomens: Das Wahrgenommene verändert sich, wenn ich es nur flüchtig, gleichsam am Rande meines Bewusstseins wahrnehme oder mich ihm mit voller Aufmerksamkeit zuwende und es ganz genau betrachte. Das Phänomen hat aber auch einen noematischen Kern, d. h. einen Gehalt, der im Wahrgenommenen selbst liegt und auch mittels noetischer Variationen - dem Versuch, das Phänomen anders zu sehen - nicht verändert werden kann. Noësis und noema können nur analytisch getrennt werden, ein Phänomen bildet immer eine noetisch-noematische Einheit. Damit ist die Spaltung von Rationalismus und Empirismus überwunden, denn ein Phänomen wird immer im Zusammenspiel von Bewusstseinsleistungen (Wahrnehmungs- und Erkenntnisakten) und dem ,empirischen“ Gehalt des Perzipierten bzw. Erkannten konstituiert.

Dieser Punkt bedarf deshalb besonderer Betonung, weil sowohl Gegner der Phänomenologie als auch skeptische Freunde oft meinen, die Phänomenologie befasse sich nur mit Bewusstseinsakten. Diese bilden indes nur die noetische Seite des Phänomens, die noematische besteht im „empirischen“ Gehalt des Wahrgenommenen. Diesbezüglich besteht kein grundsätzlicher Unterschied zum Beobachterkonzept: Man perzipiert etwas Beobachtbares. Da Phänomene eine noetisch-noematische Einheit bilden, liegen stets beide Seiten im Blickfeld der phänomenologischen Analyse. Mit der Methode der eidetischen Reduktion schlug Husserl ein Verfahren vor, das erlauben soll, das eidos, das „Wesen“ der Phänomene zu enthüllen. Mit- 
hilfe der Technik freier Variation sollen die Bestandteile, die Beschaffenheit und die Umgebung eines wahrgenommenen Phänomens so lange variiert werden, bis sich seine invarianten Eigenschaften herausschälen. Wir nehmen beispielsweise einen Tisch wahr und fragen, was das „Wesen“ eines Tischs ausmacht: Ist es immer noch ein Tisch, wenn er sechs, fünf, vier, drei, zwei Beine oder nur eines hat; wenn er aus Holz, Glas, Marmor, Plastic ist; wenn er rot, grün, gelb, blau oder weiß ist; wenn er groß oder klein ist; wenn er lang oder kurz, rechteckig, fünfeckig, dreieckig oder rund ist; etc. Auf diese Weise wird die „Idee“ des Tisches freigelegt und von seinen variablen Eigenschaften geschieden - jener Idee, die uns erlaubt, auch einen neuartigen Tisch, den wir in seinem Sosein noch nie angetroffen haben, zielsicher als „Tisch“ zu erkennen. Diese Technik ist zweifellos geeignet, verschiedene Sinnschichten eines Phänomens freizulegen, ob es hingegen sinnvoll ist, dabei platonische Ideen zugrunde zu legen, darf füglich bezweifelt werden. Im Unterschied zu Husserl hat Schütz diese Art „Bilderbuchphänomenologie“ dezidiert als Irrweg bezeichnet und die eidetischen Untersuchungen von Sozialbeziehungen, der Gemeinschaft und des Staates durch Husserls Schülerinnen Edith Stein und Gerda Walther entsprechend kritisiert (vgl. Schütz 2009, S. 305 f.). Schütz erblickte den Nutzen der Phänomenologie vielmehr in grundlegenden Konstitutionsanalysen der Lebenswelt, ähnlich dem späten Husserl (vgl. Husserl 1954).

Im Unterschied zum soziologischen Beobachterkonzept sind der phänomenologischen Analyse auch Phänomene zugänglich, die empirisch nicht beobachtbar, also intersubjektiv nicht überprüfbar sind: Träume, Fantasien, Gedanken, Absichten, Pläne, usw. Um alle diese Phänomene auf die gleiche Ebene zu stellen, schlug Husserl die Methode der transzendentalen Reduktion vor: Sämtliche ontologischen Setzungen des Common-sense sollen - nicht bestritten, aber - eingeklammert werden; diese Einklammerungen nannte er epoché. Die Phänomene sollten nicht nach ihrem ontologischen Status, sondern nach ihrer Konstitutionsweise unterschieden werden. Mit der epoché der transzendentalphänomenologischen Einstellung gewinnt auch das ego einen anderen Charakter, der nicht mit einem mundanen ego, einem in der Alltagswelt agierenden Menschen verwechselt werden darf; bei der Transzendentalphänomenologie geht es immer um die Frage nach den Bedingungen der Möglichkeit von Erkenntnis. Auch die transzendentalphänomenologische Analyse fragt aber - wie die eidetische Analyse - nach dem Sinn der Phänomene, nicht nur nach der Perzeption von Farben und Formen. In der Kategorie des Sinns erblickte Schütz denn auch die Schlüsselkategorie, um die Phänomenologie mit Webers Handlungstheorie zu verbinden und um den sinnhaften Aufbau der sozialen Welt zu analysieren.

Schütz (vgl. Schütz 2004a [1932]) wandte sich schon früh von der Transzendentalphänomenologie ab und entwickelte eine Mundanphänomenologie. Auf dic Gründe komme ich im nächsten Kapitel zu sprechen. Schütz war allerdings überzeugt, dass Husserls Erkenntnisse aus der transzendentalphänomenologischen Sphäre auch in der mundanphänomenologischen Sphäre Gültigkeit haben (was von den phänomenologischen Soziologen akzeptiert, von phänomenologischen Philosophen aber zuweilen bestritten wird). Mittels kräftiger Anleihen bei Husserl analysiert Schütz den Erlebnisstrom in seiner Zeitlichkeit, mit der Urimpression und deren retentionalen und protentionalen Abschattungen, unterscheidet die vorprädikative Sphäre der passiven Synthese von Erlebnissen von der prädikativen Sphäre der Urteile und beschreibt, wie sich Erfahrungen in Akten reflexiver Zuwendung konstituieren. Er unterscheidet polythetische von monothetischen Zugriffsweisen und macht auf die unterschiedlichen Zeitspannen der in den Blick gefassten Erlebnisse in die antizipative oder retrospektive Richtung aufmerksam. Er stellt die grundlegende Bedeutung des biographiespezifischen Wissensvorrates, dessen Typik und Relevanzstruktur heraus und analysiert die verschiedenen situativen thematischen, interpretativen und Motivations-Relevanzen in ihrem Wechselspiel. Er untersucht die Prozesse der Typisierung und unterscheidet abstrakte von konkreten, anonyme von personalen oder generelle von spezifischen Typen. Er schildert, wie der Mensch in mannigfaltigen Wirklichkeiten, also in verschiedenen Sinnbereichen lebt und beschreibt einige Merkmale, wodurch sich diese unterscheiden. Er bezeichnet die alltägliche Welt des pragmatischen Handelns als ,primordiale“ Welt und beschreibt, wie Andere dank Anzeichen und Zeichen verstanden werden. Er streicht die konstitutive Bedeutung der Sprache heraus und zeigt, wie mittels Symbolen andere, nicht direkt erfahrbare (z. B. religiöse) Wirklichkeiten appräsentiert und zugänglich werden. Alle diese Analysen erfolgen primär in einer phänomenologischen Einstellung und erschließen, wie sich beim subjektiven Erleben, bei der subjektiven Wahrnehmung Sinn konstituiert und wie der Sinn von Erfahrungen und von Handlungen gebildet wird.

Wer oder was handelt in dieser Perspektive? Schütz bleibt diesbezüglich durchaus schwammig: In Anlehnung an Husserl spricht er von ego und alter ego, aber auch vom „Ich“" und vom „Anderen“, vom „Menschen“, vom „Einzelnen“, vom „Individuum“, vom „Handelnden“ und vom „Subjekt“ - oft auch in Kombinationen wie ,der sozial Handelnde“, „,das handelnde Ich“ oder ,die psycho-physische Subjektivität ,Mensch““. Auch in der Phänomenologie bleiben viele, selbst zentrale Begriffe „operativ“, d. h. sie werden nicht als „thematische“ Begriffe erläutert und präzisiert (um eine Unterscheidung von Eugen Fink (1959) zu verwenden). Schütz untersucht auch nicht genauer, inwiefern sich das ego der transzendentalphänomenologischen Sphäre vom ego der mundanphänomenologischen Sphäre unterscheidet und was für Sinnmodifikationen es bei diesem Übergang erfährt; eher beiläufig erwähnt er, dass ,[i]n der Welt des täglichen Lebens [...] sowohl 
das Ich als auch das Du nicht als transzendentale, sondern als psychophysische Subjekte auftreten" (Schütz 2004a, S. 225). Der Subjektbegriff ist allerdings - wie im Übrigen auch die anderen aufgeführten Begriffe - Lebewesen vorbehalten, die mit Bewusstsein ausgestattet sind, und diese haben daher nicht nur einen Körper, sondern einen Leib (sie existieren als Leib).

Schütz revidierte Webers Unterscheidung von sinnhaftem Handeln und sinnlosem Verhalten. Hat man einmal die Zeitstruktur des Sinnbegriffs verstanden, so zeigt sich, dass weder Handeln noch Verhalten im Moment ihrer Ausführung sinnhaft sind. Handeln erhält seinen Sinn durch seine Vorentworfenheit modo futuri exacti, also durch den prospektiven Entwurf, Verhalten dagegen wird retrospektiv als sinnhaft wahrgenommen. Ebenso versucht Schütz die Dialektik von Strukturzwang und Handlungsautonomie, also von sozialen Bedingungen und voluntativer Handlungsabsicht durch die Zeitstruktur zu fassen: die sozialen Bedingungen bilden die Weil-Motive des Handelns, die Um-zu-Motive bestehen in den Zielen und Plänen des Handelnden. Ein konkretes Handeln wird nicht nur von selbstgewählten Motiven geleitet, sondern auch von zahlreichen auferlegten Relevanzen. Im Gegensatz zu den meisten Rational Choice-Modellen (mit Ausnahme von Esser (vgl. Esse 1991a, b)) beginnt Schütz die Analyse des Wahlhandelns nicht beim Prozess des Entscheidens zwischen Optionen (auf der Basis vorgegebener Präferenzen), sondern bei der Frage: Wie kommt es eigentlich, dass überhaupt etwas zur Wahl steht? Aus einem komplexen Spiel der petites perceptions (auch auf Gedankenebene: die Assoziationen spielen wie wild) bilden sich wohlgeformte Alternativen. Dabei unterscheidet Schütz sorgfältig zwischen der Wahl zwischen Gegenständen und der Wahl zwischen Handlungsalternativen. Haben sich solche Optionen jedoch einmal herauskristallisiert, tritt in Anlehnung an William James ein ,,voluntatives fiat“ hinzu, das aus Optionen eine Absicht generiert, die umgesetzt werden soll. Verfügten die Akteure nicht über ein Mindestmaß an Wahlfreiheit, ließe sich ihr Handeln deterministisch voraussagen (vgl. Schütz 2004b, S. 251-300).

Von besonderer Bedeutung ist die phänomenologische Einsicht, dass Akteure sich immer auf dem unbefragten Boden der Lebenswelt bewegen. Wir bewegen uns in einem Meer sinnhaft vorkonstituierter Selbstverständlichkeiten, die den Horizont dessen bilden, was uns thematisch wird oder problematisch erscheint. Auch beim Wahlhandeln wird daher unterschieden zwischen offenen und problematischen Möglichkeiten. Dies ist m. E. eine sehr zentrale Einsicht, deren Relevanz oft übersehen wird. Nur auf dieser Grundlage kann die Alltagswelt, können alltagsweltliche Interaktionen, Kommunikationen und Kollaborationen überhaupt funktionieren. $\mathrm{Zu}$ diesen Selbstverständlichkeiten gehören auch die Routinehandlungen. In seiner Analyse der Rationalität wissenschaftlicher Handlungsmodelle und der Rationalität alltagsweltlicher Handlungen hebt Schütz hervor, dass deren Ratio- nalität umso schwieriger aufzuklären sei, je standardisierter sie seien (vgl. Schütz 2010, S. 364 f.). (Dies nennt Schütz ,das Paradox der Rationalität auf der Ebene der Alltagserfahrung“", Schütz 2010). Phänomenologisch wurde dies aufgeklärt mit dem Aufweis, dass auch Routinehandlungen einmal erlernt werden mussten in Form von einzelnen, sequenziellen Schritten und polythetischen Akten der Sinnkonstitution, bevor sie inkorporiert und als routinisierte Handlungsabläufe abgespult werden konnten. Routinehandlungen können, gerade wenn sie habitualisiert und körperlich gut eingespielt sind, gleichzeitig auch mit anderen Tätigkeiten gekoppelt werden: Beherrscht man einmal das Lenken eines Autos und hat Erfahrung mit den verschiedensten Verkehrssituationen, kann man nebenher auch über etwas nachdenken, mit jemand Anderem telefonieren oder das Radio bedienen (was allerdings infolge ,beeinträchtigter Konzentrationsfähigkeit“ auch zuweilen zu Unfällen führen kann). Doch wer handelt hier eigentlich? Gibt es gleichsam mehrere egos, die nebeneinander her tätig sind: das eine intentional aufs Verkehrsgeschehen gerichtet, das andere aufs Telefongespräch und das dritte noch mit Nachdenken? Auch Routinehandlungen wie Autolenken erfordern ein Mindestmaß an intentionaler Zuwendung, werden aber zuweilen derart ,automatisch“ vollzogen, dass sie gleichsam vom Körper ausgeführt werden und das Wachbewusstsein frei für Anderes ist.

Nach phänomenologischer Auffassung ist ein mit Bewusstsein begabtes Subjekt untrennbar mit einem Leib verbunden, und zweifellos sind auch bei multiplen Tätigkeiten, die nebeneinander herlaufen, das Bewusstsein wie der Leib stets mitbeteiligt. Insofern kann man vereinfachend wohl weiterhin vom ego sprechen, auch wenn dieses ego im einzelnen nicht so klar fassbar ist. Wichtiger ist wohl der Umstand, dass das subjektive Erleben und die subjektive Erfahrung der phänomenologischen Analyse direkt zugänglich sind. Schütz ist daher der Ansicht, dass niemand so gut den subjektiven Sinn einer Handlung erfassen kann wie der Handelnde selbst. Ihm sind alle seine Erlebnisse, Erfahrungen, seine Wissensbestände, seine Handlungsziele, seine auferlegten und selbstgewählten Relevanzen in voller Fülle zugänglich. Unserem subjektiven Erleben sind Töne, Gerüche, Geräusche, Gefühle und Empfindungen zugänglich, selbst wenn wir sie nicht in sprachliche Formen gießen und verbal ausdrücken können. Wir erleben ganz unmittelbar, dass unser Erleben vorsprachliche Schichten aufweist und dass wir sinnlich erlebbare Phänomene auch in einer vorprädikativen Sphäre als sinnhaft wahrnehmen. Wir erleben oft Empfindungen und Gefühlszustände, die wir nicht sprachlich ausdrücken und kommunizieren können. Wir wissen, dass das was wir sagen, oft nicht das trifft, was wir eigentlich meinen. Und wir wissen, dass die Soziologie lange einen Bogen um Phänomene gemacht hat, die nicht leicht vertextet werden können: Bilder, Sounds, Gerüche u.v.a.m. Denn die Sozialwissenschaft ist eine Text- 
wissenschaft (vgl. Gross 1981). Die subjektive Erfahrung ist, mit anderen Worten, eine hervorragende Ressource - und jedem ist sein subjektives Erleben in direktem Zugriff möglich. Jeder lebt in seiner subjektiven Erlebnis- und Erfahrungswelt wie kommt es, dass es Soziologen gibt, die darauf verzichten wollen?

\section{Intersubjektive Verständigung}

Das Ziel von Schütz' Lebensweltanalyse war es, die sozialwissenschaftliche Methodologie philosophisch zu begründen. Die Strukturen der Lebenswelt können gelesen werden als (Proto-)Theorie des Verstehens. Nicht sich selbst zu verstehen, war sein primäres Anliegen, sondern die Frage, wie man den Anderen und dessen Handlungen verstehen und wie man sozialwissenschaftliche Konstruktionen bilden kann, die zu den alltäglichen Konstruktionen der Handelnden sinnadäquat sind. Die Gegebenheitsweise der subjektiven Erlebniswelt des Anderen ist aber eine andere als meine eigene: Während ich direkten Zugriff auf meine Erlebnisse und Erfahrungen habe, kann ich jene eines Anderen nur indirekt, nämlich signitiv vermittelt erschließen. Meine eigene Erlebnis- und Erfahrungswelt benutze ich dabei als Verstehensressource: Ich verstehe den Schmerz des anderen unter anderem, weil ich selbst schon Schmerzen erlebt habe.

Zunächst soll indes kurz erörtert werden, warum Schütz die transzendentalphänomenologische Sphäre verließ und eine Mundanphänomenologie entwickelte. Der Grund liegt darin, dass Schütz von Husserls Versuch, die Intersubjektivität transzendentalphilosophisch zu begründen, nicht überzeugt war. Im Sinnhaften Aufbau postuliert er daher die "Generalthesis des alter ego“. Das heißt, er setzte die Existenz des Anderen einfach als gegeben voraus, hoffte zu diesem Zeitpunkt aber noch, dass Husserls transzendentalphänomenologischer Begründungsversuch gelingen könnte. Später - lange nach seiner ,pragmatischen Wende“ (vgl. Srubar 1988) - begrub er diese Hoffnung und argumentierte: „Denn da menschliche Wesen von Müttern geboren und nicht in Retorten zusammengebraut werden, ist die Erfahrung der Existenz anderer menschlicher Wesen und des Sinns ihres Handelns gewiß die erste und ursprünglichste empirische Beobachtung, die der Mensch macht" (Schütz 2010, S. 455).

Es ist nicht ganz klar, welche ontologischen Annahmen des Commen-sense Schütz mit der Mundanphänomenologie übernahm: Geht es nur um den ,naiven Realismus" bezüglich der Existenz anderer Menschen - als Gegenwehr gegen den Verdacht des Solipsismus - oder geht es auch noch um weitere, in der mundanen Sphäre des Common-sense verankerte ontologische Setzungen? Mit der Mundanphänomenologie vollzog Schütz nämlich eine interessante Wendung, nämlich jene zum empirischen Subjekt: dem Menschen in seiner relativ-natürlichen Einstellung (Scheler). An ontologischen Setzungen wählte Schütz eine minimalistische Variante: „dass auch das Du Bewusstsein überhaupt habe, dass es dauere, dass sein Erlebnisstrom die gleichen Urformen aufweise wie der meine" (Schütz 2004a, S. 220). Die letztere Annahme ist konstitutiv für die Phänomenologie: Konstitutionsanalysen machen nur dann wirklich Sinn, wenn ihre Ergebnisse anthropologisch universal sind.

Obwohl Husserl die phänomenologische Analyse bei der Intentionalität des subjektiven Bewusstseins ansetzte, hatte er keinerlei solipsistische Neigungen. Den Schlüssel lieferte Leibniz' Monadologie, der auch Schütz zunächst folgte: Wenn jedes transzendentale Bewusstsein konstitutionsanalytisch dieselben formaIen Strukturen besitzt, muss das Interface analysiert werden. Auf der Ebene der Mundanphänomenologie heißt dieses Kommunikation. Schütz untersuchte primär den Prototyp der face-to-face Interaktion in leiblicher Kopräsenz und betrachtete alle anderen Interaktionsformen als davon abgeleitet. Im Unterschied zu anderen wissenssoziologischen Ansätzen wird Intersubjektivität aber nicht als gegeben betrachtet - z. B. aufgrund eines gemeinsamen Symbolsystems - sondern wird in situ hergestellt. Zeichen haben nicht nur eine objektive und situative (bei Husserl „okkasionale“) Bedeutung, sondern auch eine subjektive. Je nach Lebensgeschichte, biografiespezifischem Wissensvorrat, Typik und Relevanzsystem werden dieselben Begriffe anders gedeutet und Aussagen unterschiedlich ausgelegt. Intersubjektive Verständigung muss erarbeitet werden. Im Sinnhaften Aufbau fasst Schütz dies relativ radikal: Das Verstehen des alter ego ist nur approximativ möglich.

Nach der pragmatischen Wende von Schütz kommt allerdings eine zusätzliche Dimension hinzu: Der Verstehensprozess ist nicht nur ein Interpretationsakt des Verstehenden auf Basis seiner Selbstdeutung. In der Interaktionssituation reagiert der Andere vielmehr auf meine Deutungen, zumindest insofern sie kommunikativ erkennbar gemacht werden - oder wie Garfinkel es ausdrückte: insofern sie ,accountable“ sind. In der face-to-face Interaktion erhalte ich als Akteur also unmittelbare Feedbacks zu meinen Interpretationen, indem der Andere auf eine bestimmte Art und Weise reagiert. Kommunikative Akte sind in diesem Sinne „Wirkhandlungen“, indem sie auf das Gegenüber einen Effekt haben - und dessen interaktive Akte wiederum eine Wirkung auf mich. Schütz beschrieb diesen reziproken Wirkungszusammenhang über die Motivstruktur: Mein Um-zu-Motiv wird zum Weil-Motiv des Anderen, et vice versa.

Ein persistentes Missverständnis, das sich immer wieder mal selbst bei Kollegen äußert, die der Phänomenologie wohlgesinnt oder wenigstens nicht abgeneigt gegenüberstehen, ist folgendes: Dass sich ein Interaktionsgeschehen nach Auffassung der Phänomenologen auf die Intentionen der Akteure zurückführen 
ließe. Dies ist natürlich blanker Unsinn. Niemand kann voraussehen, wie sich ein Interaktionsablauf oder ein längeres Gespräch turn-by-turn entwickelt, welche Wendungen es nimmt und wie man vom Hundertsten ins Tausendste gelangt - es sei denn beim Spezialfall von exakt festgelegten (und befolgten) Ritualen und Zeremonien. Die phänomenologischen Soziologen sind lediglich der Ansicht, dass die Intentionalitäten der Akteure immer beteiligt sind, ja beteiligt sein müssen. Damit sind auch Perzeptionen und Imaginationen gemeint, nicht nur Um-zu-Motive, d. h. der Begriff „Intention“ darf nicht auf „Absicht" reduziert werden. Und selbstverständlich ist kein phänomenologischer Soziologe so naiv anzunehmen, dass Handlungen oder Interaktionssequenzen realiter so ablaufen, wie man sich das ursprünglich vorgestellt hat. Das hieße gerade die Zeitlichkeit außer Acht lassen, auf die die Phänomenologen so viel Wert legen: Pläne, Ziele, Vorstellungen - die im Alltag sowieso oft nur im Modus der Vagheit vorliegen - werden laufend revidiert, wenn man sich den konkreten, situativen Kontingenzen realer Handlungskontexte gegenübersieht.

Wer oder was handelt? Das Handeln wird unverbrüchlich den Akteuren zugeschrieben. Auch wenn sich diese zu Handlungs- und Verhaltensweisen hinreißen lassen, über die sie sich nachträglich selbst wundern: ohne die beteiligten Akteure hätten sie nie stattgefunden. Dies impliziert nicht, dass Akteure stets die Autoren ihrer Handlungen seien; manchmal verliert man die Beherrschung, lässt sich in einen Streit verwickeln, von Anderen zu etwas verführen. Behauptet wird lediglich ihre Beteiligung - und die Intentionalitäten der Akteure bilden dazu die Voraussetzung.

Interaktionsverläufe weisen allerdings oft auch Merkmale auf, die ein Muster interaktiven Zusammenwirkens erkennbar machen und eine Emergenzperspektive nahelegen. In der Tat hat die systemische - nicht zu verwechseln mit der systemtheoretischen - Betrachtungsweise typische interaktive Schuldzuweisungs- und Eskalationsmuster aufgezeigt, die einen unbestreitbaren Erkenntniswert besitzen. Allerdings sind solche Prozessstrukturen von Menschen produziert und sagen mehr über Dynamiken aus, in die sich Akteure mit bestimmten Perzeptions- und Deutungsmustern verwickeln, als über emergente Manifestationen eines selbstoperierenden Systems.

\section{Sozialität}

Ein Nachteil des transzendentalphänomenologischen Ansatzes ist es, dass die Analyse stets aus einem Erwachsenenbewusstsein heraus vorgenommen wird, dessen Genese nur partiell in den Blick gerät. Schütz' Hinwendung zur Mundanphäno- menologie, vor allem aber seine pragmatische Wende ermöglichte die Erkenntnis des sozio-kulturellen Apriori: Menschen werden in eine historisch gewachsene und kulturell vorgeformte Lebenswelt hineingeboren, wachsen in dieser heran und werden von ihr nachhaltig geprägt. Die sprachliche Typik, die kulturellen Muster und die gesellschaftlichen Strukturen liegen bereits vor, wenn ein Baby auf die Welt kommt; sie werden von den Heranwachsenden in ihrem Dasein und Sosein als selbstverständliche Gegebenheiten wahrgenommen. Die kollektiven Muster bilden eine unverzichtbare Grundlage für jegliche intersubjektive Verständigung: making sense funktioniert dann am besten, wenn man mit einem gemeinsamen Sprachverständnis und mit denselben kulturellen Hintergrundannahmen operiert wie das Gegenüber. (Wie gemeinsam diese im einzelnen sind, stellt sich jedoch erst sukzessive im Bemühen um interaktive Verständigung heraus.)

Schütz hat diesen Sachverhalt schon früh erkannt und seine Lebensweltanalyse daher nicht nur aus einer subjektiven Perspektive, sondern auch aus einer pragmatischen Perspektive vorgenommen. Schütz' Konzeption von Lebenswelt enthält, wie Srubar (vgl. Srubar 1988) klar herausgearbeitet hat, einen subjektiven als auch einen intersubjektiven, pragmatischen Pol. Dies impliziert, die vorhandenen kollektiven Muster zu erkennen, sie aber nicht als objektiv gegebene Bedeutungsstruktur zu reifizieren: Gesellschaftliche Wissensbestände, sprachliche Typen, kulturelle Muster müssen immer erst subjektiv angeeignet werden und erhalten dadurch spezifische Konnotationen, die - im Kontext einer spezifischen Biographie entstanden - als „subjektiv“ bezeichnet werden. Missachtet man diese, ist man für den einen Pol der Lebenswelt blind.

Vor diesem Hintergrund habe ich persönlich nie nachvollziehen können, mit welcher Begeisterung respektive Rigidität gewisse Soziologen sich allein mit „kollektiven Phänomenen“ beschäftigen wollen. Sie laufen stets Gefahr, objektive Strukturen zu reifizieren und den damit verbunden subjektiven Sinn zu übersehen - obwohl sie als erkennende Subjekte doch auch selbst einen unmittelbaren $\mathrm{Zu}$ gang zu dieser Seite haben. Es mutet oft ziemlich absurd an, wie sehr sie sich im Konzept objektiver Strukturen verheddern und dann doch irgendwie mit dem „Problem der ,agency“" fertig werden müssen. Nachdem der Mensch konzeptionell aus ihrer Soziologie verabschiedet wurde, findet sich Agency entsprechend nur noch auf einer relativ abstrakten, in jedem Fall aber auch diffusen Ebene.

\section{Die Endoxa des Common-sense}

Walter-Busch (vgl. Walter-Busch 1989) hat sich eingehend mit der Geschichte der Sozialwissenschaften und der Entwicklung ihrer Erklärungskraft beschäftigt. In Bezug auf die Managementwissenschaften, deren Aussagen sich immer mit dem 
Sachverstand praktisch handelnder und ihre Alltagspraxis reflektierender Manager messen mussten, kam er zum Schluss, dass sich der Überlegenheitsanspruch der Sozialwissenschaften nicht rechtfertigen lässt. Vielmehr habe sich im Laufe des 20. Jahrhunderts immer mehr herauskristallisiert, dass die zunehmende Verwissenschaftlichung der Managementwissenschaften nicht zu „besseren“ Einsichten führe. Die wissenschaftlichen Aussagen über die Praxis des Management seien zwar immer praxisferner und theorielastiger geworden, seien indes bei einer Rückübersetzung in die Praxis dem Erfahrungswissen der Praktiker oft unterlegen. Dass sozialwissenschaftliche Aussagen immer einen Bedeutungsüberschuss bzw. ein Begründungsdefizit aufweisen, führt er auf das Messproblem, das Erklärungsproblem und das Wertproblem der Sozialwissenschaften zurück. Darin liegt der Grund, warum die szientifische Forschungsstrategie der zunehmenden Modell- und Datenpräzisierung zu keinem kumulativen Erkenntnisfortschritt geführt hat. Vielmehr sind auch sozialwissenschaftliche Aussagen endoxa und daher von beschränkter Allgemeingültigkeit; sie sagen „viel aber Ungenaues“. Nach WalterBusch gehören Endoxa stets gesellschaftlichen Entstehungs- und Verwendungskontexten an und stehen damit in einem sozialhistorisch rekonstruierbaren Verweisungszusammenhang (vgl. Walter-Busch 1989, S. 12-14). Ihre Aussagen sind zwar methodisch kontrolliert, aber den Endoxa des Common-sense (den ,vorfindlichen Meinungen“) nicht unbedingt überlegen, manchmal - wie die Managementpraxis zeigt - auch deutlich unterlegen. (Wobei „Praxistauglichkeit“ hier nicht zu einem allgemeingültigen Kriterium wissenschaftlicher Aussagen generalisiert werden soll...)

Die zunehmende Verwissenschaftlichung manifestiert sich nicht nur bei den von Walter-Busch untersuchten Managementwissenschaften, sondern in jedem gesellschaftlichen Wissensbereich. In vielen Forschungsbereichen zeigt sich, dass die Sozialwissenschaften immer abstrakter werden, bezüglich ihrer Konzepte auch immer öfter Anleihen bei Philosophen machen und sich vom Commonsense zunehmend entfernen. Ein in Deutschland besonders populäres Beispiel ist die Systemtheorie Luhmanns, dem es gelungen ist, die gängigen Endoxa des Alltagsverstandes, die üblichen metaphysischen Setzungen außer Kraft zu setzen und Gesellschaft nochmals grundsätzlich neu zu denken. Dic Faszination dieser teilweise akrobatischen theoriebautechnischen Konstruktionen ist mir persönlich keineswegs fremd: Sich hier hineinzudenken kann durchaus Spaß machen. Die Rückübertragung der gewonnenen Erkenntnisse ins Alltagsleben - und damit ihre Sinnadäquanz - blieben mir indes ziemlich zweifelhaft.

In Bezug auf die Frage, wer oder was handelt, halte ich es für lohnenswert, sich zwischenhinein auch mal auf die Endoxa des Common-sense zu besinnen. Wir finden viele von ihnen beispielsweise in Gerichtsverhandlungen, bei denen strafrecht- liche Fälle auf der Basis nicht nur der juristischen Normen, sondern insbesondere auch des „gesunden Menschenverstands“ gefällt werden. Stets wird die Annahme zugrunde gelegt, dass Menschen nicht einfach nur Spielbälle deterministischer gesellschaftlicher Kräfte sind, sondern ihr Handeln und Verhalten auch steuern können (was wir in unserem Alltagsleben alle auch selbst erleben). Es wird aber auch über verminderte Zurechnungs- und Schuldfähigkeit verhandelt und selbstverschuldete (z. B. Angetrunkenheit) von nicht selbstverschuldeter verminderter Urteilskraft (z. B. Hirnschlag) differenziert. Man unterscheidet auch unbewusste von bewussten Motiven, echte von vorgeschobenen Motiven, absichtsvolle Mordanschläge von fahrlässigen Tötungen, und spricht insbesondere auch von Tötungen im Affekt. Man weiß auch, dass Zeugenaussagen sehr verschieden ausfallen können, die Wahrnehmung von Sachverhalten also subjektiv unterschiedlich sein kann, und dass sich Menschen oft nicht exakt erinnern können. Oft können sie das Erlebte auch nicht gut verbalisieren. Oft revidieren Betroffene später ihre vormaligen Darstellungen, und zwar nicht nur aus strategischen Gründen, sondern weil sie während des weiteren Nachdenkens und Rückbesinnens zu anderen, ,zutreffende ren" Versionen gelangen.

In Max Webers handlungstheoretischer Grundlegung der Soziologie finden sich viele dieser Endoxa wieder (vgl. Weber 1972, S. 1-10); dies vielleicht auch aufgrund seiner juristischen Ausbildung. Auch bei Schütz - womöglich ebenfalls wegen seines juristischen Hintergrunds - findet sich vieles davon wieder, wenn auch oft eher implizit. Viele dieser Prämissen kann man auch bei der konkreten Applikation qualitativer Forschungsmethoden entdecken - oft aber lediglich in vortheoretischer Form und nicht in Form einer systematischen theoretischen Grundlegung oder Methodologie. Ebenso oft vermisst man sie aber auch in der qualitativen Sozialforschung: Die verbreiteten Verfahren der akribischen Transkription von Interviews und der exakten Analyse der schriftlich fixierten Daten etwa verleihen den Aussagen eine Objektivität, die der Alltagsverstand zu relativieren weißschließlich handelt es sich um situativ erzeugte Äußerungen, die oft eher tentativen Charakter haben, die der Interviewte anschließend überdenkt, um zu treffenderen Aussagen zu kommen. Gerade diese Chance des weitergeführten Gesprächs, die im Alltag selbstverständlich ist, bleibt ihnen aber oft verwehrt.

\section{Fazit}

In diesem Beitrag versuchte ich zu skizzieren, wie die Frage ,Wer oder was handelt?“" aus der Perspektive einer phänomenologischen Soziologie beantwortet wird. Dabei versuchte ich auch einige selbst bei Wissenssoziologen verbreitete 
Missverständnisse auszuräumen und einige Unterschiede zu anderen wissenssoziologischen Ansätzen deutlich zu machen. Ich fasse im Folgenden die relevantesten Punkte nochmals in prägnanter Form zusammen:

- Wie bei Max Weber ist es der Akteur, der handelt. Schütz übernimmt die implizierte ontologische Setzung.

- Wie bei Max Weber bildet die Analyse des subjektiven Sinns, den der Handelnde mit seinem Handeln verbindet, einen zentralen Bezugspunkt der Analyse. An diesem Bezugspunkt orientiert sich auch das Postulat, sozialwissenschaftliche Konstruktionen am Kriterium der Sinnadäquanz zu beurteilen (vgl. Eberle 1999).

- Zu bedenken gilt es dabei, dass Schütz im Unterschied zu Weber den „subjektiven Sinn“ nicht in einem neukantianischen, sondern in einem phänomenologischen Rahmen auslegt.

- Schütz präzisiert die Sinnkategorie und arbeitet die Sinnstrukturen der Sozialwelt heraus. Insbesondere wird Sinn in seiner Zeitlichkeit betrachtet und subjektiver Sinn durch strikte Bezugnahme auf das erkennende Subjekt bzw. den Akteur. Die Lebenswelt hat aber nicht nur einen subjektiven, sondern auch einen intersubjektiven, pragmatischen Pol.

- Im Gegensatz zu Ansätzen sprachsoziologischer Provenienz unterscheidet die Phänomenologie eine vor-prädikative Sinnebene. Diese ist zwar selbst in der phänomenologischen Analyse schwierig zu versprachlichen, aber die vorsprachliche Ebene bildet in jedem Fall eine wichtige Schicht lebensweltlicher Sinnorientierung. Obwohl die enorme Relevanz sprachlicher Typen explizit anerkannt und betont wird, ist der Prozess der Typisierung bereits auf vorsprachlicher Ebene angelegt.

- Intentionen mit Um-zu-Motiven gleichzusetzen, ist eine Verkürzung. Die Intentionalität des Akteurs ist wesentlich umfassender als seine Um-zu-Motive und umfasst insbesondere auch das gesamte Wahrnehmungsspektrum, das durch thematische und interpretative Relevanzen und Typisierungen strukturiert wird.

- Um-zu-Motive werden immer im zeitlichen Fluss gesehen: Kein phänomenologischer Soziologe geht davon aus, dass Handlungs- oder Interaktionsverläufe einem vorentworfenen Plan folgen; vielmehr verflechten sich Um-zu- und Weil-Motive von Interaktionspartnern in einer vielschichtigen Art und verändern sich im Laufe eines Interaktionszusammenhangs. Es wäre völlig verfehlt, ein Interaktionsmuster auf einen subjektiven Plan „im Bewusstsein des einzelnen Akteurs" zurückführen zu wollen. (Dies ist leider ein verbreitetes Missverständnis bei Kritikern der phänomenologischen Soziologie.) Jedes Vorhaben, jeder Handlungsentwurf muss auch mit den Kontingenzen der jeweiligen Situation in ihrem zeitlichen Verlauf kompatibilisiert werden.
- Die Opposition verschiedener Ansätze gegen die Kategorie des Bewusstseins (Ethnomethodologie, Actor-Network-Theory) beruht wohl ebenfalls auf der Annahme, dass die Phänomenologie dem Bewusstsein einen zentralen Stellenwert zuschreibe. Sie übersehen in der Regel, dass es den Phänomenologen primär um die Intentionalität des Bewusstseins geht, also um die noetisch-noematische Einheit, oder anders ausgedrückt: um die Frage, wie sich die Welt im subjektiven Bewusstseins spiegelt - oder treffender formuliert: konstituiert.

- In Interaktionsverläufen, besonders auch in sozialen Netzwerken wie online social media, manifestieren sich zuweilen Strukturen und Prozessdynamiken, die man als ,emergent“ bezeichnen mag. Von Emergenz zu sprechen, verdeckt aber gleichzeitig den konstitutiven Zusammenhang, da diese Manifestationen nur dank der Handlungen der beteiligten Akteure überhaupt zustande kommen. Theorien der Emergenz verdecken daher gerade die zentrale Schicht der sozialen Wirklichkeit: die Ebene der Akteure. Ohne sie geschieht nämlich nichts - überhaupt nichts. Sie aus einer sozialwissenschaftlichen Theorie zu verabschieden, hat Folgen.

- Intersubjektivität ist, selbst bei gegebenem Symbolsystem und trotz der Sozialität, nicht gegeben, sondern muss in situ bewerkstelligt werden. Die Herstellung von Intersubjektivität ist ein sozialer Prozess in der Zeit.

- Das Konzept der Sozialität stellt zwar die (enorme) Prägung des heranwachsenden Menschen durch gesellschaftliche und kulturelle Wissensbestände und Objektivationen heraus, sein sozio-kulturelles Apriori also. Da der gesellschaftliche Wissensbestand (in Teilen) subjektiv angeeignet werden muss, erhalten diese Anteile des biografiespezifischen subjektiven Wissensvorrats stets auch subjektive Konnotationen. Erfahrungsgemäß spielen diese bei der intersubjektiven Verständigung eine wesentliche Rolle: Oft reden wir aneinander vorbei, verstehen unter denselben Begriffen Verschiedenes oder wir produzieren Miss-
verständnisse.

Warum streiten sich Sozialwissenschaftler so gerne über die Adäquanz der einze1nen Forschungsansätze? Naturwissenschaftler (wie mein Bruder) sind zuweilen erstaunt über die Heftigkeit, mit der sich Sozial- und Geisteswissenschaftler streiten können. Bei Naturwissenschaftlern seien, so mein Bruder, solche Religionskriege kaum zu finden, da man Aussagen des Anderen oft mit Fakten widerlegen könne; daher sei man auch in Bezug auf die eigenen Aussagen immer vorsichtig. Aufgrund des sozialwissenschaftlichen Begründungsdefizits haben die Sozialwissenschaftler demgegenüber weit mehr Freiraum für Religionskriege, und die Diagnose, dass es dabei vor allem um einen Streit über Endoxa geht, um „vorfindliche Meinungen“ also, ist kaum von der Hand zu weisen. Die Kraft der Schützschen Lebensweltanalyse liegt unter anderem auch darin, dass er die Alltagswelt und 
den Common-sense als Konstruktionen erster Ordnung und somit als Grundlage der wissenschaftlichen Konstruktionen zweiter Ordnung ausgewiesen hat. Selbst Sozialwissenschaftler, die sich kaum um die Sinnadäquanz ihrer Konzepte kümmern, haben somit die Möglichkeit, wieder ,auf den Boden der Realität" zurückzukehren. Auch wenn sie im Theoriebereich von der „Emergenz sozialer Systeme“ sprechen, kehren sie in ihrem eigenen Alltagsleben jeweils unweigerlich wieder in die Welt des Alltagsverstandes zurück, wo sie auch in ihrer Selbstwahrnehmung als Akteure handeln. Nicht „was" handelt, ist daher die Frage, sondern ,wer“ handelt: leibhaftige Akteure.
Schütz, A., \& Luckmann, T. (1975, 1984, 2003). Strukturen der Lebenswelt (Bd. 1 und 2). Konstanz: UVK (UTB).

Srubar, I (1988). Kosmion. Die Genese der pragmatischen Lebenswelttheorie von Alfred Schütz und ihr anthropologischer Hintergrund. Frankfurt a. M.: Suhrkamp.

Walter-Busch, E. (1989). Das Auge der Firma. Stuttgart: Ferdinand Enke.

Weber, M. (1972). Wirtschaft und Gesellschaft. Grundriss der Verstehenden Soziologie (5. überarbeitet Aufl. v. J. Winckelmann, Studienausgabe). Tübingen: Mohr (Paul Siebeck).

\section{Literatur}

Durkheim, E. (1970). Die Regeln der soziologischen Methode (Hrsg. und eingeleitet von René König). Neuwied: Luchterhand.

Eberle, T. S. (1999). Sinnadäquanz und Kausaladäquanz bei Max Weber und Alfred Schütz

In R. Hitzler, J. Reichertz, \& N. Schröer (Hrsg.), Hermeneutische Wissenssoziologie (S. 97-119). Konstanz: UVK.

Esser, H. (1991a). Alltagshandeln und Verstehen. Zum Verhältnis von erklärender und verstehender Soziologie am Beispiel von Alfred Schütz und Rational Choice. Tübingen: Mohr.

Esser, H. (1991b). Die Rationalität des Alltagshandelns. Eine Rekonstruktion der Handlungstheorie von Alfred Schütz. Zeitschrift für Soziologie, 20(6), 430-445.

Fink, E. (1959). Les concepts opératoires dans la phénomenologie de Husserl (S. 214-230). Paris: Les éditions de Minuit.

Gross, P. (1981). Ist die Sozialwissenschaft eine Textwissenschaft? In P. Winkler (Hrsg), Methoden der Analyse von Face-to-Face Situationen (S. 143-167). Stuttgart: J.B. Metzlersche Verlagsbuchhandlung.

Husserl, E. (1950). Cartesianische Meditationen und Pariser Vorträge (Hrsg. und eingeleitet von S. Strasser, Husserliana. Gesammelte Werke Bd. 1 (aufgrund des Nachlasses veröffentlicht vom Husserl Archiv [Louvain] unter Leitung von H. L. Breda)). Den Haag: Martinus Nijhoff.

Husserl, E. (1952). Ideen zu einer reinen Phänomenologie und phänomenologischen Philosophie (Hrsg. v. W. Biemel, Husserliana, Bd. 3 und 4). Den Haag: Martinus Nijhoff.

Husserl, E. (1954). Die Krisis der Europäischen Wissenschaften und die transzendentale Phänomenologie. Eine Einleitung in die Phänomenologische Philosophie (Hrsg. v. W. Biemel, Husserliana, Bd. 6). Den Haag: Martinus Nijhoff.

Schütz, A. (2004a). Der Sinnhafte Aufbau der sozialen Welt (ASW Bd. II. Hrsg. v. M. EndreB und J. Renn). Konstanz: UVK.

Schütz, A. (2004b). Relevanz und Handeln 1 (ASW. Bd. VI.1. Hrsg. v. E. List, unter Mitarbeit von C. Schmeja-Herzog). Konstanz: UVK.

Schütz, A. (2009). Philosophisch-phänomenologische Schriften 1 (ASW Bd. 3.1. Hrsg. v. G. Sebald, nach Vorarbeiten von R. Grathoff, T. Michael). Konstanz: UVK.

Schütz, A. (2010). Zur Methodologie der Sozialwissenschaften (ASW Bd. IV. Hrsg. v. T. S. Eberle, J. Dreher, G. Sebald, unter Mitarbeit von M. Walter). Konstanz: UVK. 
Wissen, Kommunikation und Gesellschaft

Schriften zur Wissenssoziologie

Zum Subjektverständnis der hermeneutischen Wissenssoziologie 
Wissenssoziologie hat sich schon immer mit der Beziehung zwischen Gesellschaft(en), dem in diesen verwendeten Wissen, seiner Verteilung und der Kommunikation (über) dieses Wissen(s) befasst. Damit ist auch die kommunikative Konstruktion von wissenschaftlichem Wissen Gegenstand wissenssoziologischer Reflexion. Das Projekt der Wissenssoziologie besteht in der Abklärung des Wissens durch exemplarische Re- und Dekonstruktionen gesellschaftlicher Wirklichkeitskonstruktionen. Die daraus resultierende Programmatik fungiert als Rahmen-Idee der Reihe. In dieser sollen die verschiedenen Strömungen wissenssoziologischer Reflexion zu Wort kommen: Konzeptionelle Überlegungen stehen neben exemplarischen Fallstudien und historische Rekonstruktionen neben zeitdiagnostischen Analysen.

Weitere Bände in dieser Reihe

http://www.springer.com/series/12130
Angelika Poferl $•$ Norbert Schröer (Hrsg.)

\section{Wer oder was handelt?}

\author{
Zum Subjektverständnis der \\ hermeneutischen Wissenssoziologie
}

\section{Springer VS}




\section{Inhaltsverzeichnis}

Hochschule Fulda

Fulda

Deutschland

Wissen, Kommunikation und Gesellschaft

ISBN 978-3-658-02520-5

ISBN 978-3-658-02521-2 (eBook)

DOI $10.1007 / 978-3-658-02521-2$

Dic Deutsche Nationalbibliothek verzeichnet diese Publikation in der Deutschen Nationalbibliografie; detaillierte bibliografische Daten sind im Internet über http://dnb.d-nb.de abrufbar.

Springer VS

(C) Springer Fachmedien Wiesbaden 2014

Das Werk einschließlich aller seiner Teile ist urheberrechtlich geschützt. Jede Verwertung, die nicht ausdrücklich vom Urheberrechtsgesetz zugelassen ist, bedarf der vorherigen Zustimmung des Verlags. Das gilt insbesondere für Vervielfältigungen, Bearbeitungen, Übersetzungen, Mikroverfilmungen und die Einspeicherung und Verarbeitung in elektronischen Systemen.

verfilmungen und die Einspeicherung und Verarbeitung in elektronischen Systemen.
Dic Wiedergabe von Gebrauchsnamen, Handelsnamen, Warenbezeichnungen usw. in diesem Dic Wiedergabe von Gebrauchsnamen, Handelsnamen, Warenbezeichnungen usw. in diesem
Werk berechtigt auch ohne besondere Kennzeichnung nicht zu der Annahme, dass solche Namen im Sinne der Warenzeichen- und Markenschutz-Gesetzgebung als frei zu betrachten wären und daher von jedermann benutzt werden dürften.

Der Verlag, die Autoren und dic Herausgeber gehen davon aus, dass die Angaben und Informationen in diesem Werk zum Zeitpunkt der Veröffentlichung vollständig und korrckt sind. Weder der Verlag noch die Autoren oder die Herausgeber übernehmen, ausdrücklich oder implizit, Gewähr für den Inhalt des Werkes, etwaige Fehler oder Äußcrungen.

Lektorat: Cori Antonia Mackrodt, Katharina Gonsior

Gedruckt auf säurefreiem und chlorfrei gebleichtem Papier

Springer Fachmedien Wiesbaden ist Teil der Fachverlagsgruppe Springer Science + Business Media (www.springer.com)
Wer oder was handelt? Zum Subjektverständnis der

hermeneutischen Wissenssoziologie. Eime Einleitung . . . . . . . . . .

Angelika Poferl und Norbert Schröer

Zwischen Selbstmythisierung und Entmythologisierung.

Metamorphosen des abendländischen Ichs

Hans-Georg Soeffner

Das Subjekt des kommunikativen Handelns

Hubert Knoblauch

Warum sollte die hermeneutische Wissenssoziologie an der

Rekonstruktion des subjektiven Sinns festhalten?

Norbert Schröer

Assoziationen. Über Subjektprobleme des Poststrukturalismus

und die Perspektive der Wissenssoziologischen Diskursanalyse

Reiner Kelle

Von Menschen und Dingen. Wer handelt hier eigentlich?

Jo Reichertz

Ist der Mensch ein Subjekt? Ist das Subjekt ein Mensch?

Über Diskrepanzen zwischen Doxa und Episteme

Ronald Hitzler 
Subjektive Erfahrung, intersubjektive Verständigung

und Sozialität. Phänomenologische Erörterungen

Thomas S. Eberle

Der systemtheoretische Sinn für Subjektivität

Achim Brosziewski

Kosmopolitische Empathie: Subjektivität und die fluiden

Grenzen der Sozialwelt

Angelika Poferl

\section{Subalterne Subjektivität? Zur kollektiven Handlungs-}

und Diskursfähigkeit von Dominierten .

Johannes Kniffki und Dariuš Zifonun

\section{Zur Soziologie des Zeitzeugen: Erinnerung zwischen}

Subjektivität, Sozialität und kommunikativer Konstruktion

Bernt Schnettler und Alejandro Baer

Was das Subjekt über seine Kompetenz wissen kann

Michaela Pfadenhauer

Individuum - Individualismus - Individualisierung.

Rationalität - Rationalismus - Rationalisierung.

Verstehen - Verstehende Soziale Arbeit - Verstehende

Soziale Arbeit verstehen . . . . . . . . . . . . . . . . . . . . . . 259

Ronald Kurt

\section{Autorenverzeichnis}

Alejandro Baer ist Professor für Soziologie und Direktor des Center for Holocaust and Genocide Studies an der University of Minnesota https://www.soc.umn.edu/people/baer_a.html.

Achim Brosziewski ist Professor für Bildungsforschung an der Pädagogischen Hochschule Thurgau

http://profil.phtg.ch/achim.brosziewski.

Thomas Eberle ist Professor am und Co-Direktor des Research Institute of Sociology an der School of Humanities and Social Sciences der Universität St. Gallen http://www.alexandria.unisg.ch/Personen/Person/E/Thomas_Eberle.

Ronald Hitzler ist Professor für Allgemeine Soziologie an der Fakultät für Erziehungswissenschaft und Soziologie sowie an der Fakultät für Wirtschafts- und Sozialwissenschaften der Technischen Universität Dortmund http://www.hitzler-soziologie.de.

Reiner Keller ist Professor für Soziologie an der Philosophisch-Sozialwissenschaftlichen Fakultät der Universität Augsburg

http://www.philso.uni-augsburg.de/soziologie/sozio6/Team/Keller_Reiner/.

Johannes Kniffki ist Professor für Theorien und Methoden der Internationalen Sozialen Arbeit an der Alice Salomon Hochschule Berlin

http://www.ash-berlin.eu/hs1/kniffki.

Hubert Knoblauch ist Professor für Allgemeine Soziologie an der Technischen Universität Berlin

http://www.tu-berlin.de/?id=73120. 
Ronald Kurt ist Professor für Soziologie am Fachbereich I Soziale Arbeit, Bildung und Diakonie der Evangelischen Fachhochschule Rheinland-Westfalen-Lippe in Bochum

http://www.efh-bochum.de/hochschule/lehrende detail.php?recordID=143.

Michaela Pfadenhauer ist Professorin für Kultur und Wissen am Institut für Soziologie der Universität Wien

http://www.pfadenhauer-soziologie.de.

Angelika Poferl ist Professorin für Soziologie mit Schwerpunkt Globalisierung am Fachbereich Sozial- und Kulturwissenschaften der Hochschule Fulda http://www.hs-fulda.de/poferl.

Jo Reichertz ist Professor für Kommunikationswissenschaft an der Universität Duisburg-Essen, Campus Essen

http://www.uni-due.de/kowi/JReichertz.shtml

Bernt Schnettler ist Professor für Kultur- und Religionssoziologie an der Kulturwissenschaftlichen Fakultät der Universität Bayreuth

http://www.soz.uni-bayreuth.de.

Norbert Schröer ist Professor für Qualitative Methoden der Empirischen Sozialforschung mit dem Schwerpunkt Interkulturelle Kommunikationsforschung am Fachbereich Sozial- und Kulturwissenschaften der Hochschule Fulda http://www.hs-fulda.de/schroer

Hans-Georg Soeffner ist Professor em. für Allgemeine Soziologie zuletzt an der Universität Konstanz sowie Permanent Fellow und Vorstandsmitglied am Kulturwissenschaftlichen Institut (KWI) in Essen

http://www.kwi-nrw.de/home/profil-hsoeffner.html.

Dariuš Zifonun ist Professor ist Professor für Soziologie mit dem Schwerpunkt Soziale Ungleichheit an der Alice Salomon Hochschule in Berlin http://www.ash-berlin.eu/hsl/zifonun.

\section{Wer oder was handelt? Zum Subjektverständnis der hermeneutischen Wissenssoziologie. Eine Einleitung}

\author{
Angelika Poferl und Norbert Schröer
}

\section{Wie tragfähig ist das Subjektverständnis der hermeneutischen Wissenssoziologie heute noch?}

Die hermeneutische Wissenssoziologie entwickelte sich in den vergangenen 25 Jahren zu einem eigenständigen theoretischen, methodologischen und methodischen Ansatz der gegenwärtigen sozialwissenschaftlichen Gesellschaftsanalyse. Ihre - seit den späten 1980er und 1990er Jahren - ausformulierten Beiträge haben die sozialwissenschaftliche Grundlagendiskussion aufgegriffen und auf spezifische Weise dazu beigetragen, die neuere (qualitativ und rekonstruktiv verfahrende) empirische Sozialforschung auszudifferenzieren (vgl. Hitzler 1988; Honer 1993; Schröer 1994; Knoblauch 1995; Hitzler und Honer 1997; Hitzler et al. 1999; Eberle 2000; Reichertz 2003; Keller 2005, Keller et al. 2013; grundlegend vor allem Soeffner 1989). Die Aktualität der hermeneutischen Wissenssoziologie lässt sich an vier zentralen Frage- und Erkenntnisinteressen festmachen: Bearbeitet und reflektiert wird

A. Poferl $(\bowtie) \cdot N$. Schröer

Fulda, Deutschland

E-Mail: angelika.poferl@sk.hs-fulda.de

N. Schröer

E-Mail: norbert.schroer@sk.hs-fulda.de

(C) Springer Fachmedien Wiesbaden 2014

A. Poferl, N. Schröer (Hrsg.), Wer oder was handelt?,

Wissen, Kommunikation und Gesellschaft, DOI 10.1007/978-3-658-02521-2 